\title{
Nonresonant, Femtosecond Laser Vaporization and Electrospray Post- ionization Mass Spectrometry as a Tool for Biological Tissue Imaging
}

\author{
Fengjian Shi, Jieutonne J. Archer, and Robert J. Levis*
}

Department of Chemistry and Center for Advanced Photonics Research, Temple University,

1901 N. $13^{\text {th }}$ St., Philadelphia, PA 19122

*Contact Information for Corresponding Author:

Address: Department of Chemistry, Temple University, 1901 N. $13^{\text {th }}$ St., Philadelphia, PA 19122

Phone: +1 215-204-5241

Email: rjlevis@temple.edu 


\begin{abstract}
An ambient mass spectrometry imaging (MSI) source is demonstrated with both high spatial and mass resolution that enables measurement of the compositional heterogeneity within a biological tissue sample. The source is based on nonresonant, femtosecond laser electrospray mass spectrometry (LEMS) coupled to a quadrupole time-of-flight (QTOF) mass analyzer. No matrix deposition and minimal sample preparation is necessary for the source. The laser, translation stage, and mass spectrometer are synchronized and controlled using a customized user interface. Single or multiple laser shots may be applied to each pixel. A scanning rate of 2.0 second per pixel is achieved. Measurement of a patterned ink film indicates the potential of LEMS for ambient imaging with a lateral resolution of $\sim 60 \mu \mathrm{m}$. Metabolites including sugar, anthocyanins and other small metabolites were successfully mapped from plant samples without oversampling using a spot size of $60 \times 70 \mu \mathrm{m}^{2}$. Molecular identification of the detected analytes from the tissue was enabled by accurate mass measurement in conjunction with tandem mass spectrometry. Statistical analysis, non-negative matrix factorization and principle component analysis, were applied to the imaging data to extract regions with distinct and/or correlated spectral profiles.
\end{abstract}

Keywords: Femtosecond vaporization, Ambient mass spectrometry, Mass spectrometry imaging, Tissue imaging, Statistical analysis 


\section{Introduction}

Mass spectrometry imaging (MSI) can interrogate a sample in a label-free manner and provide a chemical map in two or three dimensions with excellent molecular specificity [1]. The goal of mapping the chemical composition present in biological samples has been the driving force for the rapid development of MSI in the last few decades [2, 3]. The power of MSI has been demonstrated in many biological applications, such as imaging drugs, lipids, proteins and metabolites in tissues [4, 5], mapping chemical residues in latent fingerprints [6], and discovering clinical biomarker for tumor detection [7]. Two commonly used MSI techniques, matrix-assisted laser desorption/ionization (MALDI) [8] and secondary ion mass spectrometry (SIMS) [9, 10], produce images with excellent sensitivity and spatial resolution, but typically operate under vacuum thus exposing the biological sample to non-native conditions. Note that atmospheric pressure MALDI (AP-MALDI) has been developed in which ions are generated under atmospheric pressure $[11,12]$. MALDI also requires extensive sample preparation (e.g. matrix deposition), and SIMS is restricted to analytes with relatively low molecular weight $(<1000 \mathrm{Da})[13]$

Characterizing and imaging biological samples under native conditions with minimal sample preparation did not become routine until the introduction of ambient ionization sources including desorption electrospray ionization (DESI) [14] and direct analysis in real time (DART) [15]. Ambient ionization sources with imaging capabilities have seen rapid deployment in biological and clinical imaging applications [16]. Among numerous ambient ionization sources to date, laser vaporization has been widely adopted as one of the many desorption methods due to its spatially well-defined energy deposition ability. An additional post-ionization step (e.g. electrospray ionization (ESI)) may be coupled to the laser desorption source in many laser-based 
ambient mass spectrometry (MS) techniques [17]. The combination of laser desorption with electrospray post-ionization offers several benefits, including atmospheric pressure analysis, improved analytical sensitivity, enhanced quantitative analysis, and spatially resolved analysis $[18]$.

Ambient laser desorption in conjunction with electrospray post-ionization was first demonstrated by electrospray-assisted laser desorption/ionization (ELDI) in 2005 [19]. ELDI employs a laser beam at $337 \mathrm{~nm}$ wavelength from a nanosecond UV laser to ablate samples and the method has been applied to image slices from two types of fungus, Ganoderma lucidum and Antrodia camphorate, with a spot size of approximately $100 \times 150 \mu \mathrm{m}^{2}$ [20]. Additional examples of laser desorption-ESI hybrid techniques with imaging capabilities include matrixassisted laser desorption electrospray ionization (MALDESI) [21, 22], laser ablation electrospray ionization (LAESI) [23, 24], as well as laser electrospray mass spectrometry (LEMS) [25, 26]. The main difference among these techniques is the laser parameters, in particular pulse duration and wavelength $[18,27]$. LAESI and infrared MALDESI (IR-MALDESI) use a nanosecond IR laser at $2.94 \mu \mathrm{m}$ to resonantly excite inherent water in the biological sample to induce desorption, and have been extensively employed to image plant and animal tissue samples recently. Metabolites from plant samples [24, 28, 29] as well as endogenous lipids and small metabolites from a rat brain $[29,30]$ were imaged by LAESI with a lateral resolution of $\sim 200 \mu \mathrm{m}$, though ablation spots of $30 \mu \mathrm{m}$ size have been demonstrated for single cell analysis (but not imaging) by delivering the mid-IR laser pulses with an etched optical fiber [31, 32]. The distribution of drugs, lipids, and metabolites were also mapped from sections of animal tissue at a lateral resolution of 150 to $200 \mu \mathrm{m}$ in IR-MALDESI [22, 33], and a lateral resolution of $45 \mu \mathrm{m}$ was reported using oversampling methods where the sampling step size is smaller than the laser spot size [22, 34]. 
Laser electrospray mass spectrometry (LEMS) employs an intense $\left(10^{13}-10^{14} \mathrm{~W} / \mathrm{cm}^{2}\right)$, femtosecond (60 fs) laser pulse to induce vaporization, which differs from MALDI and other ambient laser-electrospray techniques that use nanosecond pulsed lasers operating near $10^{6}$ $\mathrm{W} / \mathrm{cm}^{2}[25]$. Unlike nanosecond laser pulses where a resonance must occur in a specific matrix, nonresonant, multiphoton laser vaporization occurs in LEMS without the aid of a matrix. Studies with thermometer ions and peptides show that LEMS produces ions with internal energies comparable to those produced by conventional ESI $[35,36]$. In the LEMS experiment, samples in numerous states (liquid, dried, mixtures, tissue, etc.) have been successfully vaporized and analyzed without matrix deposition. LEMS represents a universal tool to analyze a variety of samples intact, for samples including peptides [25], lipids [37], proteins [38-40], explosives [41, 42], narcotics [26], smokeless powders [43], pharmaceuticals [26], and tissues [44, 45]. Quantitative measurement of mixtures of small molecules revealed monotonic signal response as a function of concentration suggesting the potential of LEMS for quantitative analysis [46].

Achieving high resolution analysis in the spatial and spectral domains has been a major focus for MSI sources including laser-electrospray hybrid ambient ionization methods. MSI using LEMS was previously demonstrated by mapping oxycodone deposited on a metal plate with a lateral resolution of $250 \mu \mathrm{m}$ on a custom, low-resolution orthogonal TOF mass analyzer [26]. Recently, successful analysis was achieved with a laser spot size of $\sim 75 \mu \mathrm{m}$ [39] and endogenous metabolites and lipids were directly detected from plant and animal tissue samples in LEMS [47]. However, the ability of LEMS for MSI of biological samples at $<100 \mu \mathrm{m}$ lateral resolution has not been fully investigated and the capability of performing MS/MS analysis for molecular identification has not been demonstrated by LEMS. Here we explore the application of LEMS imaging for plant tissue samples with a high-performance mass spectrometer and a high- 
resolution translation stage. The details of the imaging source including instrument design, software development, and data analysis are presented. Spatially-resolved measurements of patterned ink on a metallic substrate are presented. MSI of a leaf at a lateral resolution of $<100$ $\mu \mathrm{m}$ is presented. Finally, statistical analysis, non-negative matrix factorization and principle component analysis, is performed as a means of spectral and spatial features extraction from the large imaging dataset.

\section{Material and methods}

\subsection{Sample preparation}

A Rhodamine $6 \mathrm{G}$ dye pattern was prepared on a stainless steel plate by a red permanent marker (Sharpie, Downers Grove, IL, USA) for LEMS imaging. The pattern was created by removal of the dye using femtosecond laser ablation $(45 \mathrm{fs}, 500 \mu \mathrm{J})$ from a Ti:Sapphire laser amplifier (Coherent Inc., Santa Clara, CA, USA) coupled with a two dimensional translation stage (MLS203-1, Thorlabs, Newton, NJ, USA) on a laser ablation platform. The platform controlled by a custom LabVIEW (LabVIEW 2012, National Instruments, Austin, TX, USA) program has the option to set the laser frequency, ablation range, and a user defined ablation pattern. Single fs laser pulses with a spot size of $50 \mu \mathrm{m}$ in diameter were used at each ablation site for the ink pattern. The custom LabVIEW ablation program and a pattern file can be found in the Appendix.

Fresh pink and red New Guinea Impatiens flower petal and leaf samples were collected from plants on the Temple University campus. The flower petals and leaves were detached from the flower samples and affixed to the stainless steel sample slides using double-sided tape prior 
to analysis. The plant samples were then directly placed on the sample stage of the ion source without any other treatment.

\subsection{LEMS imaging source}

The imaging source, shown in Fig. 1, consists of a Ti:Sapphire regenerative amplifier laser (Coherent Inc., Santa Clara, CA, USA), an electrospray emitter coupled to a quadrupole time of flight (QTOF) mass spectrometer (micrOTOF-Q II, Bruker Daltonik GmbH, Bremen, Germany), and a XY translation stage (MLS203-1, Thorlabs, Newton, NJ, USA). The source is computer-controlled by a user interface developed with LabVIEW, which allows the user to monitor sampling area, set laser and stage parameters, save stage position information, and initialize/stop imaging acquisition. The QTOF mass analyzer is controlled by the manufacturer's software (micrOTOF controller 3.0, Bruker Daltonik GmbH, Bremen, Germany) and synchronized with the LabVIEW program to enable data storage during imaging acquisition. The source can be operated in either continuous raster mode to acquire line profile of the sample or in stepped mode to enable step-by-step molecular imaging.

\subsubsection{Laser vaporization}

A Ti:Sapphire oscillator (KMLabs Inc., Boulder, CO, USA) seeded the regenerative amplifier to create $60 \mathrm{fs}$ pulses centered at $800 \mathrm{~nm}$. The laser pulse was focused to a spot size of $50 \mu \mathrm{m}$ in diameter using a $10 \mathrm{~cm}$ focal length lens (Thorlabs, Newton, NJ, USA) with an incident angle of $45^{\circ}$ with respect to the sample surface, see Appendix Supplementary Material Table S1 for more laser vaporization details. The energy of the pulse was attenuated using a quarter-wave plate and a polarizing beam splitter cube (CVI Laser Optics, Albuquerque, NM,

USA) to $\sim 1 \mathrm{~mJ}$ for the majority of the imaging experiments, resulting in an intensity of $>10^{14}$ 
$\mathrm{W} / \mathrm{cm}^{2}$ at the area sampled. For line profiling analysis, the laser was operated at $10 \mathrm{~Hz}$ when the sample mounted on the translation stage was continuously raster scanned at $1 \mathrm{~mm} / \mathrm{s}$. For imaging analysis, the laser was triggered with the choice of single or multiple laser shots at each position when the sample was moved step by step.

\subsubsection{Electrospray post-ionization and mass spectrometry}

After a laser pulse, the vaporized sample material is captured, ionized, and transferred into the QTOF mass analyzer by the electrospray stream. An acidified solvent, 1:1 (v:v) methanol/water with 1\% acetic acid (Fisher Scientific, Fair Lawn, NJ, USA), was continuously pumped through the ESI emitter by a syringe pump (Harvard Apparatus, Holliston, MA, USA) at a flow rate of $3 \mu \mathrm{L} / \mathrm{min}$. The ESI ion source was operated in positive ion mode employing drying gas at $100^{\circ} \mathrm{C}$, see Appendix Supplementary Material Table S1 for more detailed parameters. The ion optics settings of the spectrometer were optimized for best performance and were kept constant during the experiments. The spectrometer was calibrated by an ESI standard sample (Sigma-Aldrich, Buchs, Switzerland) for $m / z$ 50-1000. Some of the metabolites were identified by subjecting parent ions $(\mathrm{m} / \mathrm{z} \pm 0.5)$ to collision-induced dissociation in nitrogen gas with the collision energy set between 15-30 eV in continuous raster mode. An ESI solvent mass spectrum was acquired before each laser vaporization event to allow background subtraction of solventrelated peaks.

\subsection{Mass spectrometry imaging operation}

Through the LabVIEW user interface, a VGA camera (XVP610, IBM Inc., Armonk, NY, USA) mounted above the ion source can be used to monitor the sample target and select the region of interest. The scanning parameters, such as step size, scanning direction, scanning range, 
laser frequency, number of shots per pixel, MS scan/ablation delay, ablation/next MS scan delay are selected for an imaging experiment. A flow chart and timing diagram of the imaging system is shown in Appendix Supplementary Material Fig. S1. The LabVIEW program synchronizes the stage movement, laser triggering and mass spectrometer scan to enable step-by-step sampling and simultaneous data storage. The controllers of the translation stage and laser are communicated and triggered by LabVIEW using serial communication ports during imaging. The LabVIEW program makes calls to the Windows application programming interface (API) for managing and controlling the mass spectrometer software window to enable data storage. This API scheme bypasses the requirement of component object model (COM) library for the mass spectrometer, which is usually not supplied by the manufacturer, and provides a universal solution to controlling any commercially available mass spectrometer by LabVIEW. In the imaging mode, time-resolved mass spectra were acquired for $\sim 1.4 \mathrm{~s}$ at a spectra rate of $10 \mathrm{~Hz}$ and recorded as a function of sampling position. The dwell time of each pixel was $\sim 2.0 \mathrm{~s}$ in total, which was primarily limited by the time required to fully sample the vaporized analytes to prevent carry-over between consecutive sampling pixels. As seen in the chromatogram plot of Fig. S2, the mass spectra response of the ion of interest reaches the maximum immediately after laser ablation and drops to the low percentage range in $0.9 \mathrm{~s}$. The total time to image an organ sample with a step size of $100 \mu \mathrm{m}$ between pixels for an area of $8.1 \times 2.6 \mathrm{~mm}^{2}$ typically requires approximately $1.5 \mathrm{~h}$.

\subsection{Data processing and image visualization}

A flow diagram of data analysis is displayed in Appendix Supplementary Material Fig. S3. The raw time-resolved mass spectra files (.d) of individual pixels were first converted to standard mass spectrometry format files (.mzML) by MSConvert from ProteoWizard [48] and 
then processed by a script written in Python 2.7.8 utilizing pyOpenMS library [49] (see Appendix Supplementary Material Script S1). An individual averaged spectrum (.mzML) after blank subtraction was extracted from each time-resolved spectra in the Python script. These pixelated spectra were finally combined with the position information to generate a standard MSI data file (.imzML) by imzML converter [50]. This data processing workflow has the potential to be utilized for any standard MSI raw data files with multiple MS scans to extract a single spectrum for each pixel and incorporate the spatial information afterwards to generate a standard MSI file.

The resulted imzML file can be transferred and viewed on any free, publicly available MSI visualization software, such as BioMap [51], DataCube Explorer [52], and MSiReader [53]. Here, MSiReader was chosen to visualize the large MSI dataset with high spatial and mass resolution. The presented MSI images were smoothed with a second order linear interpolation and plotted as heatmap without any contrast enhancement.

\subsection{Statistical analysis}

Non-negative matrix factorization (NMF) and principle component analysis (PCA) were applied to the MSI data using the open source Matlab-based program OmniSpect [54] and memory efficient PCA [55] for statistical analysis. The program supports input data in the imzML format and explores the entire dataset using NMF and PCA. It loads, processes, and computes the pixel-registered mass spectra to generate statistically important component images and their corresponding component spectra. The NMF and PCA component images are presented as heatmap without any interpolation or contrast enhancement.

\subsection{Safety considerations}


Lab personnel were protected from any exposed invisible laser radiation and high voltage supplies by insulating shields. Appropriate laser eye protection was worn by all lab personnel during the experiments.

\section{Results and discussion}

\subsection{Imaging an ink pattern}

To evaluate the imaging capability of the LEMS system, a red ink pattern on a stainless steel slide was used as the initial sample for study. This pattern was created by removal of the ink using femtosecond laser ablation to form a ' $\mathrm{T}$ ' with the custom laser ablation platform. An optical image of the ink pattern is shown in Fig. 2a with a dark and bright area corresponding to laser-unablated and ablated ink areas, respectively. Fig. 2c shows the ion image at $\mathrm{m} / \mathrm{z} 442.90$, corresponding to rhodamine $6 \mathrm{G}$ dye with the loss of a chloride ion, obtained by LEMS using a $50 \mu \mathrm{m}$ laser spot size (see Appendix Supplementary Material Fig. S4), which agrees with the optical image of the pattern. The variance of pixel intensity is not due to laser fluctuations, but is due to inhomogeneity inherent in the printed dye film, which has been seen in previous LEMS [37] and MALDI experiments [56]. A plot of the intensity of the dye ion as a function of position, as indicated by the white dotted line in Fig. 2c, is shown in Fig. 2d. The ion intensity of the gap area that is indicated by the white arrow in Fig. 2a, corresponding to the 'valley' in the plot, revealed a nearly zero level. The width of the 'valley' was determined to be $550 \mu \mathrm{m}$, which agrees with the expected distance based on the pre-defined ablation pattern.

To evaluate the degree of sample carry-over between pixels, the background ion signals before firing the laser are extracted from the time-resolved spectra of individual pixels and plotted in the Appendix Supplementary Material Fig. S5. As seen in Fig. S5b and S5c, the 
background ion image implies little or no sample carry-over between consecutive pixels after the $2.0 \mathrm{~s}$ dwell time and the ion image without background subtraction remains at the same intensity scale as the background subtracted image. The mean value of the lateral resolution was determined to be $62.9 \mu \mathrm{m}$, shown in Fig. 2d and Appendix Supplementary Material Fig. S6, using the distance for which an increase in signal intensity from $20 \%$ to $80 \%$ of the maximum value as a measure of the lateral resolution [10,57]. This proof-of-principle experiment of the ink pattern demonstrates that a lateral resolution of $\sim 60 \mu \mathrm{m}$ can be achieved by the current LEMS imaging system.

\subsection{Tissue mass spectrometry of flower petal and leaf}

To demonstrate the ability of LEMS for characterization of biological samples, a flower petal and leaf sample from a pink Impatiens plant were directly analyzed in continuous raster mode with a laser spot size of $60 \mu \mathrm{m}$ in diameter. The blank-subtracted mass spectra of the flower petal and leaf are presented in Fig. 3 top and bottom, respectively. In the petal spectrum, the $\mathrm{m} / \mathrm{z} 741.19$ ion was tentatively identified as protonated pelargonidin 3-(pcoumaroyl)glucoside-5-glucoside, a flavonoid pigment associated with pink/red plant tissues [58, 59], by mass searching in the METLIN metabolites database [60] (http://metlin.scripps.edu/index.php, last accessed October 7, 2015). This identification was confirmed by the fragmentation pattern using tandem mass spectrometry (MS/MS) analysis with features corresponding to the loss of glucose and glucoside coumaroyl group in Appendix Supplementary Material Fig. S7. The ions at $\mathrm{m} / \mathrm{z} 711.18$ and 783.21 were tentatively assigned to delphinidin 3,5-di-acetylglucoside and pelargonidin 3-(p-coumaryl)glucoside-5-acetylglucoside, respectively. Some of the peaks shared between the petal and leaf are at $\mathrm{m} / \mathrm{z} 287.05$ and 471.08 , which are protonated cyanidin and sodiated cyanidin glucoside, and an unidentified ion at $\mathrm{m} / \mathrm{z}$ 
587.12. Nevertheless, ions that are specific to the leaf were found, including $\mathrm{m} / \mathrm{z} 179.04$ and 161.03, which are likely an unidentified species $[\mathrm{M}]^{+}$or $[\mathrm{M}+\mathrm{H}]^{+}$and its fragment $\left[\mathrm{M}-\mathrm{H}_{2} \mathrm{O}\right]^{+}$or $\left[\mathrm{M}-\mathrm{H}_{2} \mathrm{O}+\mathrm{H}\right]^{+}$. Direct analysis of the plant samples suggest that anthocyanins, sugars and other small metabolites can be detected by LEMS without any sample preparation. Identification of the detected molecular features is possible through exact mass measurement and MS/MS analysis to provide chemical information regarding the sample.

\subsection{Tissue imaging of flower leaf}

To demonstrate the application of LEMS imaging for biological samples, a sample of red Impatiens flower leaf was placed in the ion source and directly imaged using a laser spot size of $60 \mu \mathrm{m}$ and a raster step size of $100 \mu \mathrm{m}$. A continuous raster scan profile of the sample revealed abundant features including $\mathrm{m} / \mathrm{z}$ 303.05, 449.11, 595.15, and 727.19 (see Appendix Supplementary Material Fig. S8), corresponding to delphinidin, cyanidin 5-glucoside, cyanidin 3-(p-coumaroyl)glucoside, and cyanidin 3-(p-coumaryl)sambubioside, respectively. Other ions at $\mathrm{m} / \mathrm{z} 604.38,717.47,830.56$, and 943.64 are unidentified metabolites. The same leaf sample was then imaged over an area of $8.1 \times 2.6 \mathrm{~mm}^{2}$ and the optical image of the leaf following MSI is shown in Fig. 4a with the ablation grid displayed on the sample. Selected ion images of $\mathrm{m} / \mathrm{z}$ 303.05, 717.47 and 449.11 are shown in Fig. 4b-4d, respectively. A complete list of the representative ion images is provided in the Appendix Supplementary Material Fig. S9.

The ion species at $m / z$ 717.47, as seen in the ion image in Fig. 4c, is distributed evenly throughout the entire leaf area scanned. The ion image also displays a sharp edge that is in good correlation with the margin of the leaf, as seen in the overlay image in Appendix Supplementary Material Fig. S10a. A representative line intensity plot extracted from the $\mathrm{m} / \mathrm{z} 717.47$ ion image, shown in the Appendix Supplementary Material Fig. S10b, demonstrates the signal stability 
within the sample. The variation of the signal could be caused by several factors including the amount of metabolite in the area probed, the changes of surface height from the plant morphology, and the stability of the electrospray, which had been observed in a previous nanoDESI mass spectrometry imaging experiment [61]. In contrast, the ion images of delphinidin and cyanidin glucoside, $m / z 303.05$ (Fig. 4b) and 449.11 (Fig. 4d), reveal a distribution that appears to be specific to the vein area of the leaf, as seen in the overlay image of $m / z 303.05$ with the optical image in Fig. 4f. The actual laser-ablated crater size on tissue was determined to $60 \times 70$ $\mu \mathrm{m}^{2}$, as seen in the microscope image on the left of Fig. 4e. This crater was successfully mapped on the ion image as a single pixel on the right of Fig. 4e, which corresponds to the dashed area of the sample. This experiment suggests that tissue imaging of plant samples at a lateral resolution $<100 \mu \mathrm{m}$ is possible for LEMS. Regions that presented distinct spectra profiles can be resolved by LEMS imaging.

\subsection{Statistical analysis}

The large size and high complexity of the MSI datasets make the manual examination of the selected ion maps impossible when identifying features of interest from hundreds to thousands of detected ions. Instead, statistical analysis (e.g. principle component analysis (PCA) and non-negative matrix factorization (NMF)) has been utilized in MSI for high-throughput data analysis where the results are shown not as heatmap of selected ions but as score plots in the spatial domain [62]. The use of PCA in MSI data analysis is considered to be controversial due to the generation of negative loading and score plots, which are values on the new coordinate system (PCs) and the values have no direct physical meaning, and so other statistical analysis tools such as NMF have been employed to provide more interpretable component images and spectra with non-negative factors $[55,62]$. 
Here, NMF and PCA were applied to the LEMS imaging results of the red Impatiens flower leaf sample in Fig. 4. The entire dataset was computed and reduced to different components based on the similarities of the spatial distributions. Fig. 5 displays the major component images and their corresponding component spectra by NMF and PCA analysis. In the first NMF component image (NMF1), solvent features that are suppressed inside the tissue region are classified by the NMF analysis. The second NMF component (NMF2) reveals the same leaf pattern as the selected ion images in Fig. 4. More importantly, the corresponding component spectrum displays the same ion species as those observed from the line scan spectra in Appendix Supplementary Material Fig. S8. The agreement of the component images and corresponding component spectra from NMF analysis with the results from manually selected ion maps and mass spectra indicates that NMF can be applied to the LEMS imaging dataset to extract spatial and spectral features of interest efficiently for high-throughput analysis. However, note that NMF generates only one component image that contributes to all biologically relevant ion species in NMF2. In PCA analysis, the PC1 and PC2 component images show similar results as NMF1 and NMF2 that anti-correlated distributions corresponding to plant and solvent species are distinguished. Unlike NMF analysis where plant features are grouped into only one component image, PCA, an algorithm based on finding maximum variance, resolves the unique distribution of $m / z$ 303.0, 449.1, 595.1, and 727.2 ions from the remaining plant features in the fourth principle component (PC4) images.

In an effort to optimize the statistical analysis of the test data, we removed pixels corresponding to the non-leaf area from the imaging dataset. This customized dataset was then imported to NMF and PCA analysis (see Appendix Supplementary Material Fig. S11). As seen for the NMF outputs, leaf features are classified into two components in NMF1 and NMF2, 
where the NMF2 component image reveals the unique distribution of the vein area. But the NMF2 component spectrum suggests that plant species that are also found in the non-vein area contribute to the distribution in the NMF2 component image. In contrast, these species are classified from the vein area species by the PCA analysis in the PC3 image. This example demonstrates that statistical analysis can be combined with LEMS imaging to extract features of interest from the large imaging data, and PCA is able to resolve statistically small differences that NMF has difficulty in identifying. LEMS imaging with statistical analysis has the potential to be used as an efficient diagnostic tool in biological and clinical applications, such as tumor detection and biomarker discovery.

\section{Conclusions}

The use of non-resonant fs lasers in LEMS imaging overcomes the need for resonant absorption occurring in analyte, matrix or substrate, thus making the vaporization step both universal and sample independent. The design and implementation of the LEMS imaging source on a commercial QTOF mass spectrometer is described. MSI can be performed with dwell times as low as $2.0 \mathrm{~s} / \mathrm{pixel}$ in single or multi-shot ablation with full control over the repetition rate and the delay between the ablation event and the mass spectrometer scan. A lateral resolution of 60 $\mu \mathrm{m}$ was demonstrated without oversampling on an ink pattern by LEMS. Chemically distinct features smaller than $100 \mu \mathrm{m}$ were successfully mapped by LEMS imaging on tissue samples. Efficient feature extraction from the large amount of LEMS imaging data was achieved by NMF and PCA analysis. Compositional heterogeneity within the sample was successfully distinguished when the imaging data is subjected to PCA analysis.

\section{Acknowledgements}


The authors thank Hannes L. Röst (Department of Biology, Institute of Molecular Systems Biology, ETH Zürich) for the support of pyOpenMS. The authors thank Dr. May D. Wang (Department of Biomedical Engineering, Georgia Institute of Technology) and Dr. R. Mitchell Parry (Department of Computer Science, Appalachian State University) for the assistance of using OmniSpect. Finally, the authors gratefully acknowledge the funding support from the National Science Foundation (CHE 0957694). 


\section{References}

[1] A.D. Palmer, T. Alexandrov, Anal. Chem. 87 (2015) 4055-4062.

[2] R.M.A. Heeren, Int. J. Mass Spectrom. 377 (2015) 672-680.

[3] J.L. Norris, R.M. Caprioli, Chem. Rev. 113 (2013) 2309-2342.

[4] J.M. Wiseman, D.R. Ifa, Y. Zhu, C.B. Kissinger, N.E. Manicke, P.T. Kissinger, R.G. Cooks, Proc. Natl. Acad. Sci. U.S.A. 105 (2008) 18120-18125.

[5] E.H. Seeley, R.M. Caprioli, Proc. Natl. Acad. Sci. U.S.A. 105 (2008) 18126-18131.

[6] M.J. Bailey, N.J. Bright, R.S. Croxton, S. Francese, L.S. Ferguson, S. Hinder, S. Jickells, B.J.

Jones, B.N. Jones, S.G. Kazarian, J.J. Ojeda, R.P. Webb, R. Wolstenholme, S. Bleay, Anal.

Chem. 84 (2012) 8514-8523.

[7] H. Meistermann, J.L. Norris, H.-R. Aerni, D.S. Cornett, A. Friedlein, A.R. Erskine, A.

Augustin, M.C. De Vera Mudry, S. Ruepp, L. Suter, H. Langen, R.M. Caprioli, A. Ducret, Mol. Cell. Proteomics 5 (2006) 1876-1886.

[8] R.M. Caprioli, T.B. Farmer, J. Gile, Anal. Chem. 69 (1997) 4751-4760.

[9] C.L. Brummel, I.N.W. Lee, Y. Zhou, S.J. Benkovic, N. Winograd, Science 264 (1994) 399402.

[10] T.L. Colliver, C.L. Brummel, M.L. Pacholski, F.D. Swanek, A.G. Ewing, N. Winograd, Anal. Chem. 69 (1997) 2225-2231.

[11] V.V. Laiko, A.L. Burlingame, U.S. Patent 5965884 (1999).

[12] V.V. Laiko, M.A. Baldwin, A.L. Burlingame, Anal. Chem. 72 (2000) 652-657.

[13] L.A. McDonnell, R.M.A. Heeren, Mass Spectrom. Rev. 26 (2007) 606-643.

[14] Z. Takáts, J.M. Wiseman, B. Gologan, R.G. Cooks, Science 306 (2004) 471-473.

[15] R.B. Cody, J.A. Laramée, H.D. Durst, Anal. Chem. 77 (2005) 2297-2302.

[16] C. Wu, A.L. Dill, L.S. Eberlin, R.G. Cooks, D.R. Ifa, Mass Spectrom. Rev. 32 (2013) 218243.

[17] M.E. Monge, G.A. Harris, P. Dwivedi, F.M. Fernández, Chem. Rev. 113 (2013) 2269-2308.

[18] P.M. Flanigan, R.J. Levis, Annu. Rev. Anal. Chem. 7 (2014) 229-256.

[19] J. Shiea, M.-Z. Huang, H.-J. Hsu, C.-Y. Lee, C.-H. Yuan, I. Beech, J. Sunner, Rapid Commun. Mass Spectrom. 19 (2005) 3701-3704.

[20] M.-Z. Huang, S.-C. Cheng, S.-S. Jhang, C.-C. Chou, C.-N. Cheng, J. Shiea, I.A. Popov, E.N. Nikolaev, Int. J. Mass Spectrom. 325-327 (2012) 172-182.

[21] J.S. Sampson, A.M. Hawkridge, D.C. Muddiman, J. Am. Soc. Mass Spectrom. 17 (2006) $1712-1716$.

[22] G. Robichaud, J.A. Barry, K.P. Garrard, D.C. Muddiman, J. Am. Soc. Mass Spectrom. 24 (2013) 92-100.

[23] P. Nemes, A. Vertes, Anal. Chem. 79 (2007) 8098-8106.

[24] P. Nemes, A.A. Barton, Y. Li, A. Vertes, Anal. Chem. 80 (2008) 4575-4582.

[25] J.J. Brady, E.J. Judge, R.J. Levis, Rapid Commun. Mass Spectrom. 23 (2009) 3151-3157.

[26] E.J. Judge, J.J. Brady, D. Dalton, R.J. Levis, Anal. Chem. 82 (2010) 3231-3238.

[27] G. Robichaud, J.A. Barry, D.C. Muddiman, Atmospheric Pressure Mass Spectrometry

Imaging, in: Encyclopedia of Analytical Chemistry, John Wiley \& Sons, Ltd, (2006).

[28] P. Nemes, A.A. Barton, A. Vertes, Anal. Chem. 81 (2009) 6668-6675.

[29] H. Li, B.K. Smith, L. Márk, P. Nemes, J. Nazarian, A. Vertes, Int. J. Mass Spectrom. 377 (2015) 681-689.

[30] P. Nemes, A.S. Woods, A. Vertes, Anal. Chem. 82 (2010) 982-988. 
[31] B. Shrestha, A. Vertes, Anal. Chem. 81 (2009) 8265-8271.

[32] B. Shrestha, J.M. Patt, A. Vertes, Anal. Chem. 83 (2011) 2947-2955.

[33] J.A. Barry, M.R. Groseclose, G. Robichaud, S. Castellino, D.C. Muddiman, Int. J. Mass Spectrom. 377 (2015) 448-455.

[34] G. Robichaud, J. Barry, D. Muddiman, J. Am. Soc. Mass Spectrom. 25 (2014) 319-328.

[35] P.M. Flanigan, F. Shi, J.J. Perez, S. Karki, C. Pfeiffer, C. Schafmeister, R.J. Levis, J. Am. Soc. Mass Spectrom. 25 (2014) 1572-1582.

[36] P.M. Flanigan, F. Shi, J.J. Archer, R.J. Levis, J. Am. Soc. Mass Spectrom. 26 (2015) 716724.

[37] J.J. Brady, E.J. Judge, R.J. Levis, J. Am. Soc. Mass Spectrom. 22 (2011) 762-772.

[38] E.J. Judge, J.J. Brady, R.J. Levis, Anal. Chem. 82 (2010) 10203-10207.

[39] F. Shi, P.M. Flanigan, J.J. Archer, R.J. Levis, Anal. Chem. 87 (2015) 3187-3194.

[40] S. Karki, P.M. Flanigan, J.J. Perez, J.J. Archer, R.J. Levis, J. Am. Soc. Mass Spectrom. 26

(2015) 706-715.

[41] J.J. Brady, E.J. Judge, R.J. Levis, Rapid Commun. Mass Spectrom. 24 (2010) 1659-1664.

[42] P.M. Flanigan, J.J. Brady, E.J. Judge, R.J. Levis, Anal. Chem. 83 (2011) 7115-7122.

[43] J.J. Perez, P.M. Flanigan, J.J. Brady, R.J. Levis, Anal. Chem. 85 (2013) 296-302.

[44] E.J. Judge, J.J. Brady, P.E. Barbano, R.J. Levis, Anal. Chem. 83 (2011) 2145-2151.

[45] P.M. Flanigan, L.L. Radell, J.J. Brady, R.J. Levis, Anal. Chem. 84 (2012) 6225-6232.

[46] P.M. Flanigan, J.J. Perez, S. Karki, R.J. Levis, Anal. Chem. 85 (2013) 3629-3637.

[47] F. Shi, P.M. Flanigan, J.J. Archer, R.J. Levis, J. Am. Soc. Mass Spectrom. 27 (2016) 542551.

[48] D. Kessner, M. Chambers, R. Burke, D. Agus, P. Mallick, Bioinformatics 24 (2008) 25342536.

[49] H.L. Röst, U. Schmitt, R. Aebersold, L. Malmström, Proteomics 14 (2014) 74-77.

[50] A.M. Race, I.B. Styles, J. Bunch, J. Proteomics 75 (2012) 5111-5112.

[51] M. Stoeckli, D. Staab, M. Staufenbiel, K.-H. Wiederhold, L. Signor, Anal. Biochem. 311 (2002) 33-39.

[52] I. Klinkert, K. Chughtai, S.R. Ellis, R.M.A. Heeren, Int. J. Mass Spectrom. 362 (2014) 4047.

[53] G. Robichaud, K.P. Garrard, J.A. Barry, D.C. Muddiman, J. Am. Soc. Mass Spectrom. 24 (2013) 718-721.

[54] R.M. Parry, A.S. Galhena, C.M. Gamage, R.V. Bennett, M.D. Wang, F.M. Fernández, J. Am. Soc. Mass Spectrom. 24 (2013) 646-649.

[55] A.M. Race, R.T. Steven, A.D. Palmer, I.B. Styles, J. Bunch, Anal. Chem. 85 (2013) 30713078.

[56] R.R. Hensel, R.C. King, K.G. Owens, Rapid Commun. Mass Spectrom. 11 (1997) 17851793.

[57] S.L. Luxembourg, T.H. Mize, L.A. McDonnell, R.M.A. Heeren, Anal. Chem. 76 (2004) 5339-5344.

[58] R. Freyre, C. Uzdevenes, L. Gu, K.H. Quesenberry, J. Am. Soc. Hortic. Sci. 140 (2015) 4549.

[59] F. He, N.-N. Liang, L. Mu, Q.-H. Pan, J. Wang, M.J. Reeves, C.-Q. Duan, Molecules 17 (2012) 1571-1601.

[60] C.A. Smith, G. O'Maille, E.J. Want, C. Qin, S.A. Trauger, T.R. Brandon, D.E. Custodio, R. Abagyan, G. Siuzdak, Ther. Drug Monit. 27 (2005) 747-751. 
[61] J. Laskin, B.S. Heath, P.J. Roach, L. Cazares, O.J. Semmes, Anal. Chem. 84 (2012) 141148.

[62] E.A. Jones, S.-O. Deininger, P.C.W. Hogendoorn, A.M. Deelder, L.A. McDonnell, J. Proteomics 75 (2012) 4962-4989. 


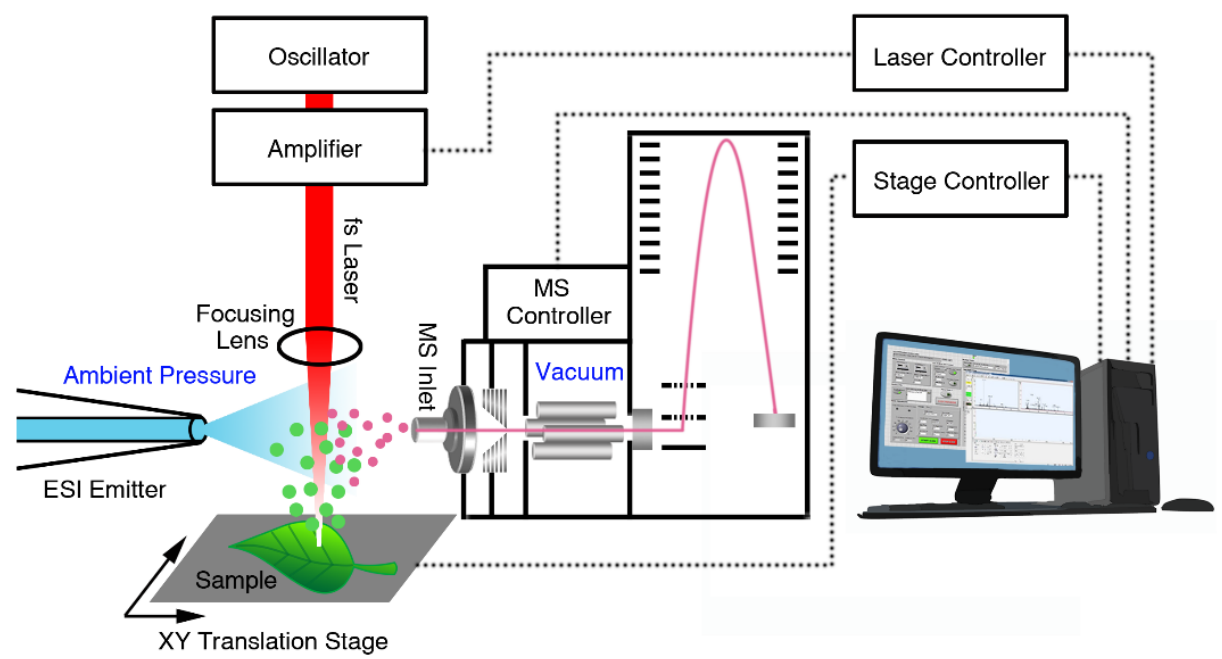

Fig. 1. Schematic of the femtosecond laser vaporization and electrospray post-ionization MSI system. Femtosecond laser pulses from a Ti:Sapphire oscillator were regeneratively amplified and focused on the sample by a lens. The sample was placed on an XY translation stage under ambient conditions and raster-scanned step by step during MSI analysis. The laser-vaporized analytes were captured and ionized by an ESI source and transferred into a QTOF mass analyzer. The synchronization of translation stage, laser, and mass spectrometer was computer-controlled by a customized user interface. 
a)

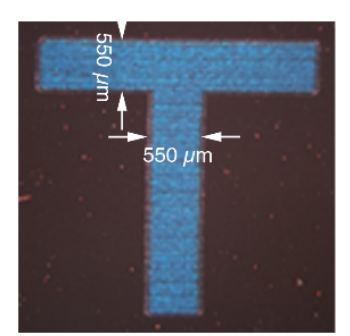

c)

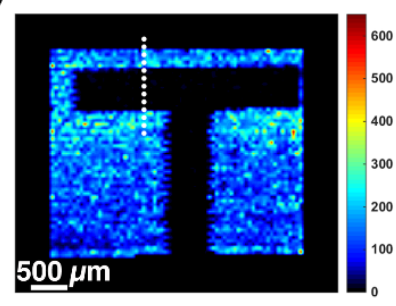

b)

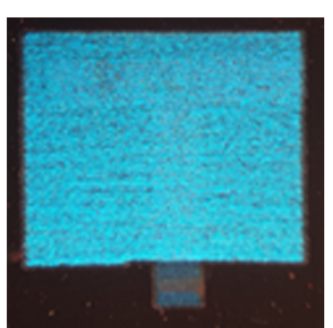

d)

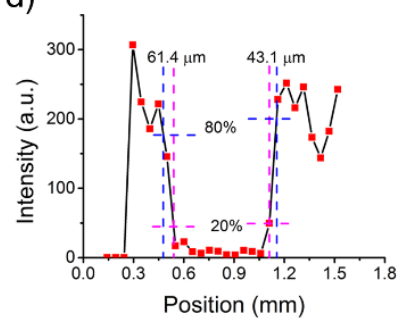

Fig. 2. Optical image of a laser-printed pattern in a thin film of red ink containing rhodamine 6G dye on a metal slide a) pre-LEMS and b) post-LEMS imaging. c) Selected ion image ( $\mathrm{m} / \mathrm{z} 442.90$ \pm 0.01 ) acquired with a single laser shot per pixel. d) Intensity plot as a function of positions, as shown by the white dotted line in c). The blue and magenta lines indicate $80 \%$ and $20 \%$ of the maximum peak intensity, respectively. 


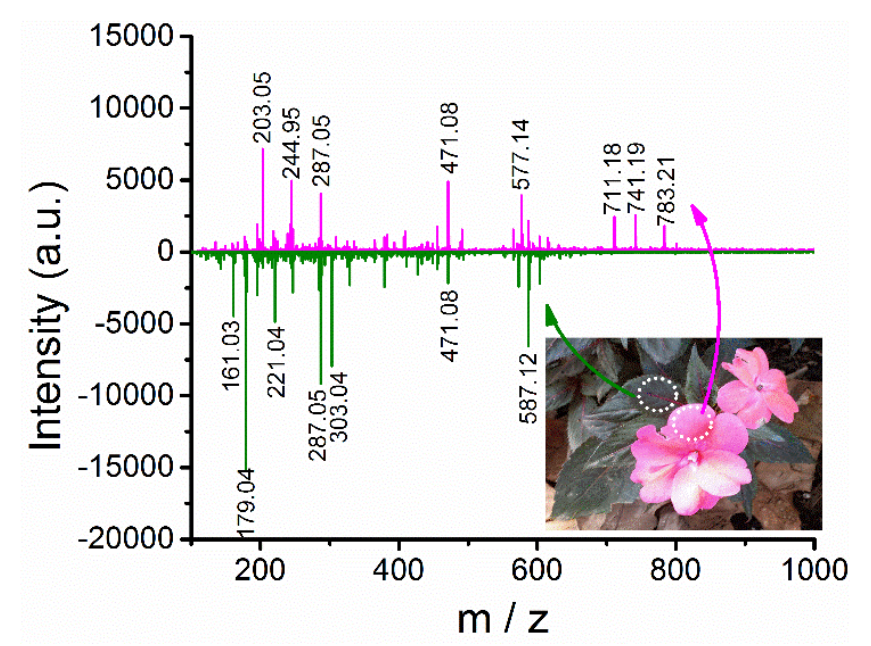

Fig. 3. Blank-subtracted LEMS mass spectra of a pink Impatiens flower petal (top) and leaf (bottom). An optical image of the flower is shown in the inset with the analyzed regions circled. 
a)

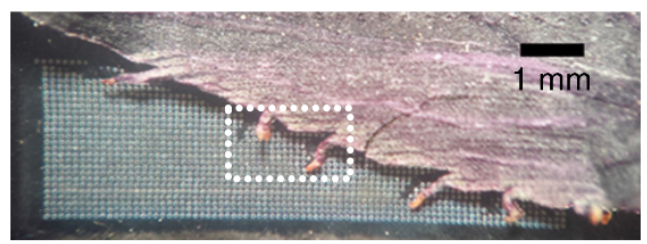

c)

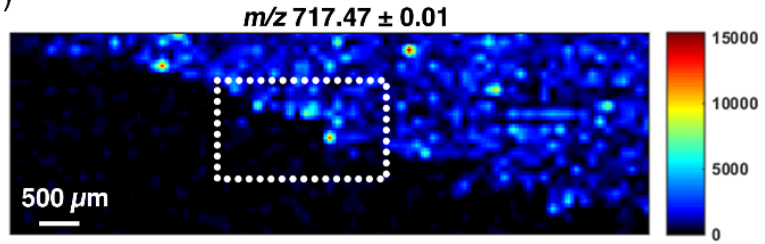

e)

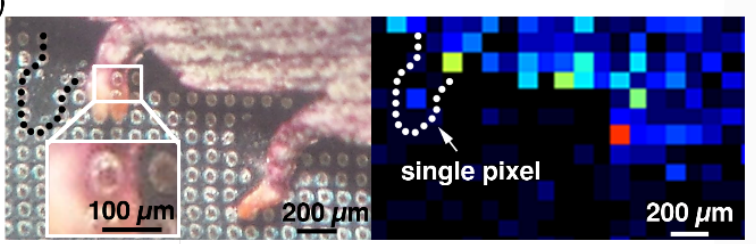

b)

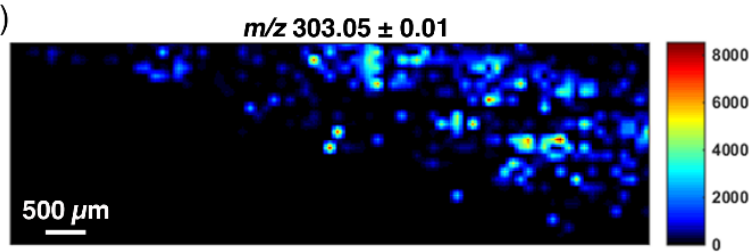

d)

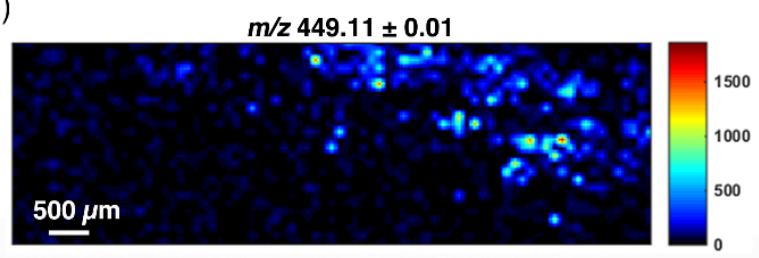

f)

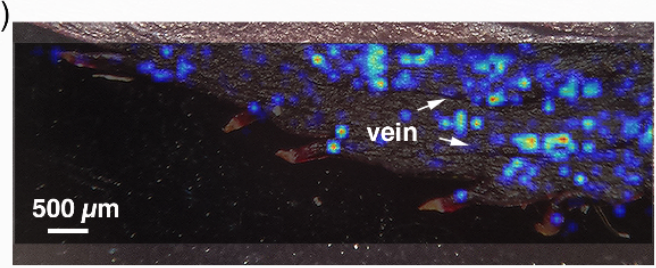

Fig. 4. a) Optical image of a red Impatiens flower leaf ablated by LEMS using a single laser shot per pixel. Mass spectrometry images showing the spatial distributions of ions at $m / z$ b) 303.05 , c) 717.47, and d) 449.11 with the absolute intensity scale of each isolated ion. e) Zoomed-in optical (left) and mass spectrometry (right) images of an area indicated by the rectangles in (a) and (c), respectively. Note that the leaf sample shifted slightly to the right with respect to the ablation grid when the optical image was taken. f) Overlay of the $m / z 303.05$ ion distribution map and the optical image. 

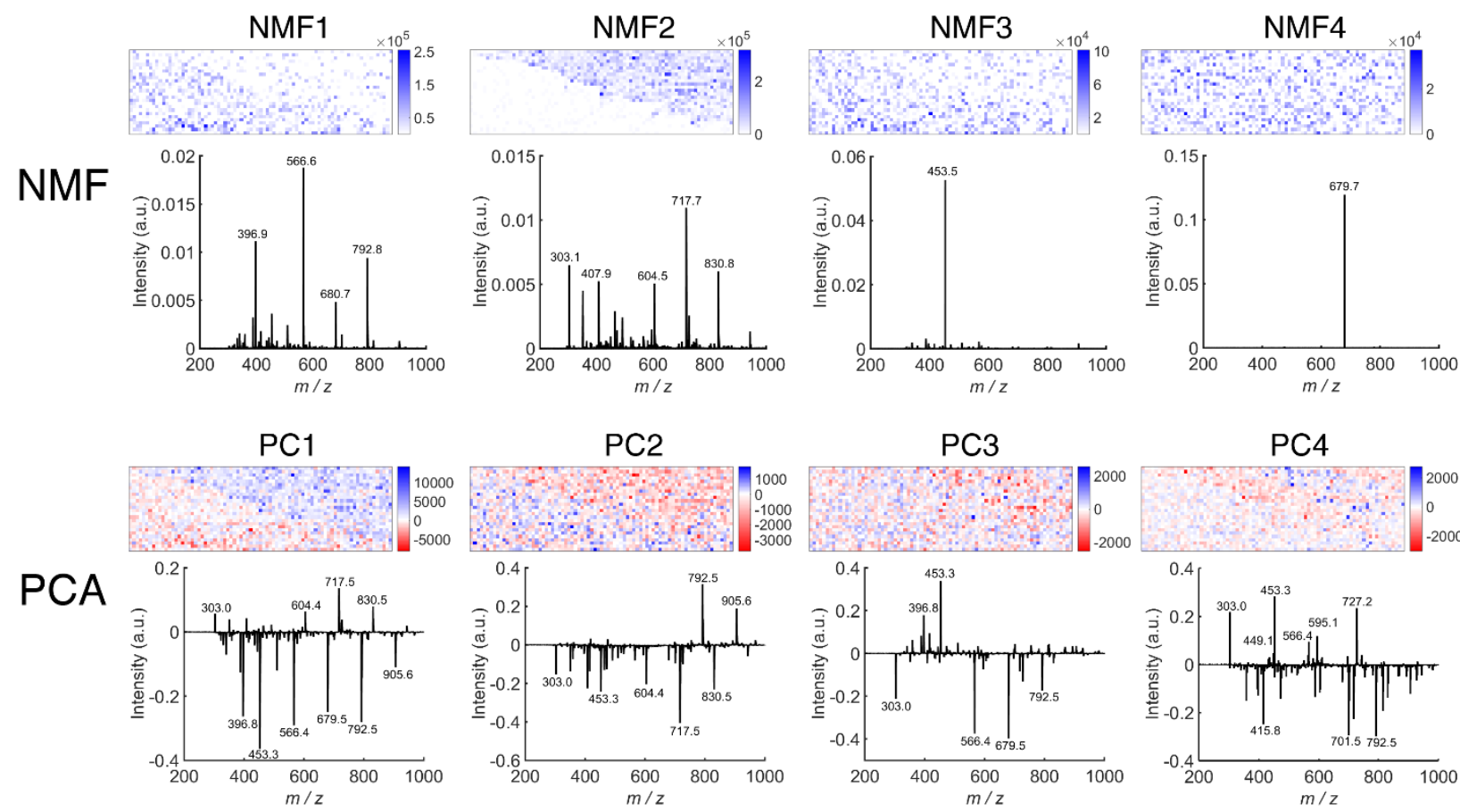

Fig. 5. Comparison of PCA and NMF outputs of the red Impatiens flower leaf sample. The component images (top) and their corresponding component spectra (bottom) are shown for PCA and NMF, respectively. 